\title{
El teónimo PTGYH de la inscripción filistea de Ecrón
}

\author{
Carlos Alonso \\ Universidad Complutense, Madrid \\ Andrés PIQUER \\ Universidad Complutense, Madrid \\ Artes serviunt vitae, sapientia imperat \\ In memoriam Antonio Peral
}

La inscripción filistea de Ecrón fue descubierta en Tel Miqne (Khirbet el- Muqanna'), Israel, al final de la temporada de excavaciones arqueológicas del verano de 1996. Este yacimiento, situado a unos $35 \mathrm{~km}$. al oeste de Jerusalén, ya había sido relacionado con el sitio de la ciudad de Ecrón por J. Naveh en $1958{ }^{1}$. La identificación propuesta entonces a partir de la descripción bíblica de las fronteras de la tribu de Judá (Jos 15:10-11 y 45-46) queda ahora confirmada por este importante texto epigráfico en el que el oferente es el príncipe o gobernante o, por un posible calco semántico del acadio ${ }^{2}$, rey de

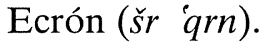

La inscripción de Ecrón consta de cinco líneas con un total de setenta y dos letras en lo que parece ser una escritura local, la cual comparte algunos rasgos con la escritura paleohebrea y otros con la fenicia. Las palabras, veintiuna en total, van separadas por un punto a

${ }^{1}$ S. Gitin, T. Dothan, J. NAVEh, «A Royal Dedicatory Inscription from Ekron», Israel Exploration Journal [=IEJ] 48 (1997) págs. 1-16; y J. NAVEH, «Khirbet el-Muqanna“ - Ekron», IEJ 8 (1958) págs. 87-100 y 165-170.

2 A las acepciones usuales de 'jefe, alto funcionario, gobernante, príncipe', etc., que tiene la palabra šr en las lenguas semíticas noroccidentales, posiblemente haya que añadir por influjo del acadio sarru $(m)$ el significado de 'rey'. Cf. J. HofTIJZER, K. Jongeling, Dictionary of the North-West Semitic Inscriptions (Leiden - New York - Köln 1995) vol. II pág. 1190; y M. E. J. RICHARDSON (ed.), The Hebrew and Aramaic Lexicon of the Old Testament ... (Leiden - New York - Köln 1994-2000) vol. III págs. 1350-1353. 
excepción de la que ocupa la última posición y la del estado constructo šr 'qrn 'rey de Ecrón'. De esas veintiuna palabras, siete son nombres propios, de los cuales uno es un topónimo (Eqrôn), otro un teónimo femenino $(P T G Y H)$, y cinco antropónimos masculinos que indican el nombre y la alcurnia del oferente de la inscripción. Cinco de las palabras son verbos de las siguientes raíces: b.n.y 'construir', b.r.k 'bendecir' (dos veces), s.m. [r] 'guardar' y 'r. $\underline{k}$ 'prolongar'. Las nueve restantes son los sustantivos $b t$ 'casa, edificio, templo', $b n$ 'hijo' (cuatro veces en el contexto de la alcurnia del oferente), šr 'príncipe, gobernante, rey', y otras tres con el pronombre personal de tercera persona masculino singular - $h$ sufijado: 'dth 'su señora', $y m h$ 'sus días' y [']rșh 'su tierra'.

El texto reza ${ }^{3}$ así:

$$
\begin{aligned}
& \text { 1. בת.בן.אכיש.בן.פדי .בן. }
\end{aligned}
$$

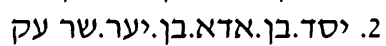

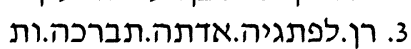

$$
\begin{aligned}
& \text { 4. שמ[ר]ה.ותארך.ימה.ותברך. } \\
& \text { (א] }
\end{aligned}
$$

1) BT.BN.'KYŠ.BN.PDY.BN

2) YSD.BN.'D'.BN.Y'R. ŠR 'Q-
Templo (que) construyó 'Akîs, hijo de Padi, hijo de

YSD, hijo de 'Ada', hijo de Ya'ir, rey de 'Eq-

3) RN.LPTGYH.'DTH.TBRKH.WT- ron, para PTGYH, su señora. Ella le bendiga y

4) ŠM[R]H.WT'RK.YMH.WTBRK. le guarde y prolongue sus días y bendiga

5) [']RṢH

su tierra.

A pesar de su brevedad y de su carácter estereotipado de fórmula de dedicación, este texto es el más extenso encontrado hasta ahora en una ciudad filistea. Gracias a las fuentes asirias podemos fechar esta inscripción en la primera mitad del siglo VII a.E.c. En efecto, el oferente, 'Akîšs, hijo de Padi, es coetáneo del rey de Asiria Asarhaddón (680-669 a.E.c.) y participa en la primera campaña contra Egipto del rey Asurbanipal en el año 667 a.E.c.; su padre, Padi, también era conocido por las fuentes asirias en relación con la campaña del 701

\footnotetext{
${ }^{3}$ El texto fue publicado y traducido por GiTIN «A Royal Dedicatory» pág. 9.
} 
a.E.c. llevada a cabo por Senaquerib, así como por una breve inscripción del mismo yacimiento ( $l b^{\prime} l$ wlpdy 'Para Báal y para Padi') ${ }^{4}$.

La lengua de la inscripción pertenece claramente al grupo semítico nor-occidental, subgrupo cananeo (scriptio defectiva, contracción de diptongos de origen *aw y *ay, y uso de un léxico muy característico para las palabras 'hijo' y 'tierra'), pero presenta dos nombres propios, los más importantes, por cierto, que no parecen ser semíticos. Uno de ellos es el del oferente 'Akîšs y el otro el de la diosa $P T G Y H$ a quien se dedica la inscripción y el templo.

En la Biblia se menciona el nombre de 'Akn̂šs, rey (o reyes) de la ciudad filistea de Gat en época del rey Saúl (1Sam 21:11-16: 'Aknî̌s, a secas; 1Sam 27:2: 'Akiš, hijo de Ma 'ôk ) y a principios del reinado de Salomón (1Reyes 2:39s: 'Akي̂šs, hijo de Ma’akâ). De ahí que vocalicemos el nombre 'KYŠ de la inscripción filistea según el texto masorético, pues ciertamente las consonantes del nombre son las mismas en el texto hebreo bíblico y en la inscripción que nos ocupa. Ahora bien la vocalización masorética, que también aparece en la Vulgata latina (Achis) y en el Targum ('Akîš), presenta algún problema, pues en las inscripciones asirias el 'Ak̂îš de Ecrón aparece como Ikausu (i-ka-u-su) y las versiones antiguas griegas del 'Akîš de Gat son: 'Ag.khoûs y 'Ak.khoûs, lo que permitiría conjeturar un posible nombre de origen indoeuropeo transcrito al filisteo por 'KYŚ y al asirio *Ikayus > Ikausu, procedente de un *Akhayus, relacionado con el griego 'Akhaiós, es decir, 'Aqueo'. Esta conjetura sería preferible a la antigua propuesta de relacionarlo con el antropónimo 'Ag.khísês 'Anquises' 5.

El teónimo femenino $P T G Y H$, desconocido hasta su aparición en este epígrafe, es particularmente interesante, pues, al contrario de otros dioses filisteos mencionados en la Biblia, no parece ser un nombre semítico. En nuestra opinión podría tratarse de un nombre indoeuropeo compuesto de dos elementos: $P T$, relacionado con la raíz *POT / POS 'señor', y GYH, que se podría relacionar con el griego gaîa o gê 'tierra' ${ }^{6}$.

${ }^{4}$ Cf. J. B. Pritchard (ed.), Ancient Near Eastern Texts Relating to the Old Testament (Princeton 1969) págs. 291 y 294, y 287s, respectivamente; y S. GITIN, M. COGAN, «A New Type of Dedicatory Inscriptión from Ekron», IEJ 49 (1999) págs. 193-202.

5 GITIN «A Royal Dedicatory» pág. 11.

${ }^{6}$ Cf. J. POKORNY, Indogermanisches Etymologisches Wörterbuch (Bern - München 1959) vol. I pág. 842: «poti-s, Hausherr, Herr, Gatte', neben -pot- im Kompositum; fem *potnî; zugrunde liegt unflektiertes poti selbst». P. CHANTRAINE, 
La interesante propuesta de A. Demsky ${ }^{7}$ de leer $P T N Y H$ y relacionar este teónimo con el griego pótnia 'señora' no es posible ni necesaria.

En primer lugar la grafía $P T G Y H$ es bastante clara. Ciertamente, en la editio princeps de este epígrafe se señala con un punto la letra $<g\rangle$, pero ello se debe a que, en palabras de los editores, aunque 'segura' (certain) es 'algo defectuosa' (somewhat defective); y nosotros suponemos que han extremado la cautela por darse la circunstancia de que no aparece en la inscripción ninguna otra $\langle g\rangle$ y esta se encuentra en una palabra desconocida. Ahora bien, la grafía $\langle n>$ sí que está abundantemente representada (seis veces) y no es posible leer $P T N Y H$. Por otra parte, la $<n>$ de pótnia no forma parte de la raíz indoeuropea, sino que se trataría de un morfema secundario de femenino, comparable a lo que ocurre en sánscrito con pati- 'señor, marido', cuyo femenino es patn $\hat{\imath}-$, y con râja- 'rey, rajá', cuyo femenino es râjnî̀- 'reina' (en aglo-indio rani). F. R. Adrados ${ }^{8}$ señala con mucha claridad que de pótis 'señor, marido' se derivaría en griego pótnia 'la del marido, esposa', que nos parece que habría que considerar en relación con un sufijo primario de pertenencia sánscrita (masc. -in-, fem. $\left.-i n \hat{\imath}^{-}\right)$.

No es, pues, necesaria la corrección, pero sí que creemos acertado retener la relación de las dos primeras consonantes $P T$ - de este teónimo con la raíz indoeuropea *POT / POS de muy amplia difusión ${ }^{9}$.

También quisieramos destacar el uso de palabras derivadas de esta raíz en algunas relaciones nominales que, sin ánimo de ser exhaustivos, daremos a continuación. Así, por ejemplo, encontramos en micénico: po-ti-ni-ja a-si-wi-ja 'señora de Asia' y po-ti-ni-ja i-qe-ja 'señora de los caballos'; en griego pótnia thêrôn 'señora de las fieras' (cf. latín potens ferarum) y pótnia gê 'señora de la tierra'; en latín potens deo-

Dictionaire étymologique de la langue grecque (Paris 1984) propone (págs. 931s) pósis masc. 'esposo y dueño de la casa' y pótnia fem. 'ama', junto con el sánscrito pátni fem. 'ama; diosa', fem. arcaico de pósis; y en relación con gaîa y gê fem. 'tierra' señala (pág. 218) que no tienen una etimología establecida.

7 A. DEMSKY, «The Name of the Goddess of Ekron: A New Reading», Journal of the Ancient Near Eastern Society 25 (1997) págs. 1-5.

${ }^{8}$ F. AdRAdos, Historia de la Lengua Griega (Madrid 1999) pág. 22.

${ }^{9}$ Cf. E. A. RoBerts, B. PASTOR, Diccionario etimológico indoeuropeo de la Lengua Española (Madrid 1996) pág. 138, donde relaciona poti- 'poderoso, señor' con sánscrito pati-, avéstico paiti-, albanés patte, letón pats, latín potis y griego despotês <*dems-pot 'amo de casa', de donde procede déspota. 
rum, potens maris, potens Cypri y potens frugum, referidos respectivamente a Jupiter señor de los dioses, Neptuno señor del mar, Venus señora de Chipre y Ceres señora de los cereales. Este uso de palabras con la raíz indoeuropea *POT / POS sería, en nuestra opinión, semejante al que nos permite interpretar el teónimo $P T G Y H$ como 'Señora de la tierra'.

A punto de cerrar este artículo nos llega la noticia de una posible interpretación en relación con el santuario de Delfos Pytho y la divinidad Gaia propuesta por C. Schäfer-Lichtenberger ${ }^{10}$. Coincidimos, al parecer, en la interpretación del segundo elemento de este nombre, pero nos parece preferible, a la vista de los usos arriba indicados y de la gran difusión de la raíz *POT / POS, la interpretación que nosotros proponemos para el primer elemento de este teónimo.

En cualquier caso y pese a las incertidumbres, la interpretación de este nuevo teónimo por medio del indoeuropeo hace aconsejable sumarlo a las «pruebas acumulativas» que, basándose en el criterio de proximidad de las transcripciones onomásticas, intentan relacionar a los filisteos y a otros de los llamados «Pueblos del Mar» con el mundo greco-micénico ${ }^{11}$.

Excepto estos dos nombres propios que, como hemos visto, parecen ser de origen indoeuropeo, la inscripción está redactada en una lengua semítica que sin duda sería la de la población local de la zona. Todo parece indicar, pues, que los descendientes de los invasores filisteos terminaron adoptando el dialecto nativo del lugar en que se asentaron sus antepasados en la primera mitad del siglo XI a.E.c. Este fenómeno, salvando las distancias, sería comparable al que tuvo lugar con los visigodos en la España romana, los francos en la Galia y los normandos en Inglaterra, por citar algunos ejemplos relativamente próximos.

\footnotetext{
${ }^{10}$ C. SCHÄFer-Lichtenberger, «PTGJH Göttin und Herrin von Ekron», Biblische Notizen 91 (1998) págs. 64-76, citado por W. W. HALlo (ed.) en The Context of Scripture (Leiden - Boston - Köln 2000) vol. II pág. 164, nota 6.

${ }^{11}$ Cf. J. Alvar, Los Pueblos del Mar y otros movimientos de pueblos a fines del Segundo Milenio (Madrid 1989) págs. 38-46.
} 


\section{RESUMEN}

La inscripción filistea de Ecrón, que fue descubierta en las excavaciones arqueológicas de Tel Miqne (Israel) del año 1996, está redactada en un dialecto cananeo, pero en ella se menciona un teónimo femenino $P T G Y H$, hasta ahora desconocido, que se ha intentado interpretar mediante las lenguas indoeuropeas. En nuestra opinión, podría tratarse de un nombre compuesto de dos elementos: $P T$ relacionado con la raíz *POT / POS 'señor' y GYH relacionado con el griego gaîa 'tierra', lo que nos llevaría a interpretar este teónimo como 'Señora de la tierra'.

\section{SUMMARY}

The Philistine Ekron inscription, discovered during the 1996 archaeological campaign at Tell Miqne (Israel), is written in a Canaanite dialect, but it mentions a feminine theonym, Ptgyh, unknown until now. Its explanation has been attempted through comparison with the Indoeuropean languages. In our opinion, it may be a name composed of two elements: $P T$, related to the root $*$ POT / POS 'Lord', and GYH 'earth'. Thus, this theonym may be understood as 'the Earth Lady'. 